\title{
3 hyperbaric oxygen treatments reduced cognitive sequelae of acute carbon monoxide poisoning
}

Weaver LK, Hopkins RO, Chan KJ, et al. Hyperbaric oxygen for acute carbon monoxide poisoning. N Engl J Med 2002;347:1057-67.

\section{QUESTION: In patients with carbon monoxide poisoning, is hyperbaric oxygen (HBO) more effective than normobaric oxygen for reducing cognitive sequelae?}

\section{Design}

Randomised (allocation concealed*), blinded (patients, outcome assessors, and statisticians),* controlled trial with follow up at 6 weeks, 6 months, and 1 year.

\section{Setting}

Emergency departments in Utah, Idaho, and Wyoming, USA.

\section{Patients}

152 patients (mean age 36 y, 62\% men) with documented or obvious exposure to carbon monoxide and any of 11 predetermined symptoms. Patients were excluded if they were $<16$ years of age, moribund, or pregnant or if $>24$ hours had elapsed since exposure. Follow up at 6 weeks was $97 \%$.

\section{Intervention}

All patients had 3 hyperbaric chamber sessions at intervals of 6-12 hours starting within 24 hours after carbon monoxide exposure. All non-intubated patients received oxygen, $15 \mathrm{l} / \mathrm{min}$ via a non-rebreathing face mask and reservoir. Intubated patients were mechanically ventilated with $100 \%$ oxygen. 76 patients were allocated to $\mathrm{HBO}$ and exposed to $100 \%$ oxygen at 3 atmospheres $(304 \mathrm{kPa})$ and then 2 atmospheres absolute $(203 \mathrm{kPa})$ during the first chamber session and 100\% oxygen at 2 atmospheres absolute for sessions 2 and 3.76 patients were allocated to normobaric oxygen and exposed to air at 1 atmosphere absolute $(101.3 \mathrm{kPa}$, or sea level pressure) for all 3 chamber sessions.

\section{Main outcome measures}

Cognitive sequelae at 6 weeks were measured using a battery of neuropsychological tests and were considered present if any subtest score was $>2$ standard deviations (SDs) below the mean of demographically corrected standardised scores or if $\geq 2$ scores were $>1$ SD below the mean.

\section{Main results}

Analysis was by intention to treat. The trial was stopped early after 3 of 4 planned interim analyses were completed. At 6 weeks, 6 months, and 12 months, patients in the $\mathrm{HBO}$ group had fewer cognitive sequelae than did patients in the normobaric group (table). The analysis was adjusted for cerebellar dysfunction because it was not equally distributed (4 $\mathrm{HBO} v 15$ normobaric group patients), with no change in results.

\section{Conclusion}

In patients with acute carbon monoxide poisoning, 3 hyperbaric oxygen treatments within 24 hours of exposure reduced cognitive sequelae at 6 weeks, 6 months, and 1 year.

*See glossary.

Source of funding:

not stated.

For correspondence: Dr L K Weaver, LD Hospital, Salt Lake City, UT, USA lweaver@ihc.com

Abstract and commentary also appear in ACP Journal Club.

Hyperbaric oxygen v normobaric oxygen for acute carbon monoxide poisoning†

\begin{tabular}{lllll}
$\begin{array}{c}\text { Neurological } \\
\text { sequelae }\end{array}$ & Hyperbaric & Normobaric & RRR (95\% CI) & NNT (Cl) \\
at 6 weeks & $25 \%$ & $46 \%$ & $46 \%(15$ to 66$)$ & 5 (3 to 18$)$ \\
\hline at 6 months & $21 \%$ & $38 \%$ & $45 \%(8.3$ to 67$)$ & $6(4$ to 39$)$ \\
\hline at 1 year & $18 \%$ & $33 \%$ & $44 \%(2.2$ to 68$)$ & 7 (4 to 182$)$ \\
\hline
\end{tabular}

†Abbreviations defined in glossary; RRR, NNT, and $\mathrm{Cl}$ calculated from data in article. Analysis includes all patients. Those with missing data at 6 weeks were assumed to have cognitive sequelae; those with cognitive sequelae at 6 weeks and missing data at 6 or 12 months were assumed to have cognitive sequelae at those time points.

\section{COMMENTARY}

Although carbon monoxide, the most frequent cause of poisoning, results in both immediate and delayed cognitive deficits, physicians have been justifiably skeptical about treating it with $\mathrm{HBO}$ rather than normobaric oxygen. $\mathrm{HBO}$ often incurs the cost and risk associated with transferring a potentially unstable patient to a referral centre, and observational studies that initially suggested a benefit were recently contradicted by a well designed but negative randomised trial by Scheinkestel $e t$ al.

The study by Weaver $e t$ al showed a large neuropsychological benefit in the HBO group. This study avoids 2 of the limitations of the study by Scheinkestel $e t$ al: loss to follow up and cluster rather than patient level randomisation for group exposures. The Scheinkestel trial also differed in other respects. More patients had attempted suicide (69\% compared with $31 \%$ in the study by Weaver et al), and depression and other toxins may have influenced outcomes. Patients in the Scheinkestel trial control group received continuous face mask oxygen at $15 \mathrm{l} / \mathrm{min}$ for 3 days, whereas patients in the study by Weaver et al received oxygen only when needed to maintain oxygen saturation. Both studies concealed allocation and used sham hyperbaric treatments to enhance blinding, and both used persistent or delayed neurological sequelae as a primary outcome. However, each used a different battery of tests. Similarly, differences in treatment delay, case severity, and $\mathrm{HBO}$ protocols might also explain the disparity between the results of these 2 studies.

The study by Weaver et al shifts the balance of evidence to support HBO over normobaric oxygen. Future studies should be designed to determine optimal combinations of the dose and timing of $\mathrm{HBO}$, and planned subgroup analyses should attempt to define which patients could benefit most from hospital transfer. Hopefully, these studies will provide the evidence needed to erase the remaining doubt about the effectiveness of $\mathrm{HBO}$

Stephen Pitts, MD, MPH Emory University Atlanta, Georgia, USA

1 Scheinkestel CD, Bailey M, Myles PS, et al. Hyperbaric or normobaric oxygen for acute carbon monoxide poisoning: a randomised controlled clinical trial. Med J Aust 1999;170:203-10. 